\title{
Helicity selection rules and noninterference for BSM amplitudes
}

\author{
Aleksandr Azatov, ${ }^{1}$ Roberto Contino, ${ }^{2,3,{ }^{*}}$ Camila S. Machado, ${ }^{4,3}$ and Francesco Riva ${ }^{3}$ \\ ${ }^{1}$ Abdus Salam International Centre for Theoretical Physics, Strada Costiera 11, 34151 Trieste, Italy \\ ${ }^{2}$ Institut de Théorie des Phénomenes Physiques, EPFL, 1015 Lausanne, Switzerland \\ ${ }^{3}$ Theoretical Physics Department, CERN, 1211 Geneva, Switzerland \\ ${ }^{4}$ Instituto de Física Teórica, Universidade Estadual Paulista, 01049-010 São Paulo, Brazil
}

(Received 26 July 2016; published 20 March 2017)

\begin{abstract}
Precision studies of scattering processes at colliders provide powerful indirect constraints on new physics. We study the helicity structure of scattering amplitudes in the standard model (SM) and in the context of an effective Lagrangian description of beyond-the-SM (BSM) dynamics. Our analysis reveals a novel set of helicity selection rules according to which, in the majority of $2 \rightarrow 2$ scattering processes at high energy, the SM and the leading BSM effects do not interfere. In such situations, the naive expectation that dimension-6 operators represent the leading BSM contribution is compromised, as corrections from dimension- 8 operators can become equally (if not more) important well within the validity of the effective field theory approach.
\end{abstract}

DOI: 10.1103/PhysRevD.95.065014

\section{INTRODUCTION}

Standard model (SM) precision tests represent an important strategy in the search for new physics. Effective field theories provide a suitable theoretical framework in which these tests can be performed model independently, while maintaining a simple connection to explicit UV theories. The effective field theory (EFT) approach is especially convenient to organize hierarchically possible departures from the SM. Models in which a large separation exists between the new physics scale $\Lambda$ and the electroweak (EW) scale can be expanded in powers of fields and derivatives, ${ }^{1}$

$\mathcal{L}=\mathcal{L}^{\mathrm{SM}}+\mathcal{L}^{6}+\mathcal{L}^{8}+\cdots, \quad \mathcal{L}^{D}=\sum_{i} c_{i}^{(D)} \mathcal{O}_{i}^{(D)}$,

where $c_{i}^{(D)} \sim \Lambda^{4-D}$ and $D$ is the dimension of the operator $\mathcal{O}_{i}^{(D)}$. In most theories, $D=6$ terms are expected to capture the leading beyond-the-SM (BSM) effects. (In the presence of approximate symmetries or other selection rules, effects from $D=6$ operators can be suppressed compared to those from $D=8$ or higher-dimensional operators; see [1,2]). This motivates searches for generic new physics, as parametrized by $\mathcal{L}^{6}$ only [3-5]. In particular, when departures from the SM are small, as typically occurs in weakly coupled theories, the leading corrections to the cross section are expected to arise

*On leave from Dipartimento di Fisica, Università di Roma La Sapienza and INFN, Roma, Italy.

${ }^{1}$ In the following we assume for simplicity that the UV dynamics conserves baryon and lepton numbers.

Published by the American Physical Society under the terms of the Creative Commons Attribution 3.0 License. Further distribution of this work must maintain attribution to the author(s) and the published article's title, journal citation, and DOI. at order $1 / \Lambda^{2}$ from the interference between the SM and $D=6$ operators. The aim of this article is to assess the validity of this naive expectation by analyzing the relative importance of the contributions to scattering amplitudes from the different terms in Eq. (1).

Precision searches can be divided into two categories: (i) those exploiting the resonantly enhanced production of a SM state (such as measurements at the $Z$-pole or single-Higgs production); and (ii) those exploiting the high-energy $E \gg m_{W}$ behavior of nonresonant processes (including $e^{+} e^{-} \rightarrow f \bar{f}$ at LEP2 and $W^{+} W^{-}$production). This second mode of exploration is ubiquitous in the LHC experimental program [4-8], as an obvious consequence of its high-energy reach, and it is the focus of this work.

We anticipate our main result in Table I: in the highenergy (massless) limit and working at tree level, SM and $D=6 \mathrm{BSM}$ contributions to $2 \rightarrow 2$ scattering processes involving at least one transversely polarized vector boson

TABLE I. Four-point amplitudes $A_{4}$ that do not vanish in the massless limit and the total helicity $h\left(A_{4}\right)$ of their SM and BSM contributions. $V=V^{ \pm}, \psi=\psi^{ \pm}$and $\phi$ denote, respectively, transversely polarized vectors, fermions (or antifermions) and scalars in the SM. For processes with at least one transversely polarized vector (listed above the double line in the table), SM and BSM contributions do not interfere in the massless limit because they have different total helicity.

\begin{tabular}{lcc}
\hline \hline$A_{4}$ & $\left|h\left(A_{4}^{\mathrm{SM}}\right)\right|$ & $\left|h\left(A_{4}^{\mathrm{BSM}}\right)\right|$ \\
\hline$V V V V$ & 0 & 4,2 \\
$V V \phi \phi$ & 0 & 2 \\
$V V \psi \psi$ & 0 & 2 \\
$V \psi \psi \psi \phi$ & 0 & 2 \\
$\psi \psi \psi \psi \psi$ & 2,0 & 2,0 \\
$\psi \psi \phi \phi$ & 0 & 0 \\
$\phi \phi \phi \phi$ & 0 & 0 \\
\hline \hline
\end{tabular}


appear in different helicity amplitudes and thus do not interfere. This noninterference rule contradicts the naive expectation and implies that in these processes $D=6$ and $D=8$ operators contribute at the same order in the $1 / \Lambda$ expansion if masses and loop corrections are neglected. It follows that in many cases of interest analyses based on an EFT truncated at the $D=6$ level are incomplete in the high-energy region away from threshold.

\section{HELICITY SELECTION RULES AND NONINTERFERENCE}

When departures from the SM are small, the leading BSM contribution comes from the SM-BSM interference term in the amplitude squared. Obviously, interference is possible only if SM and BSM give nonvanishing contribution to the same helicity amplitude. In this section we study the helicity structure of scattering amplitudes at tree level in the SM and at leading order in the effective field theory expansion, i.e. at the level of $D=6$ operators. We denote the corresponding new-physics contribution as $\mathrm{BSM}_{6}$ in the following. We focus first on the phenomenologically relevant case of $2 \rightarrow 2$ scatterings and work in the massless limit; the massive case and higher-point amplitudes are discussed below. We use the spinor-helicity formalism (see Refs. [9,10] for a review), where the fundamental objects which define the scattering amplitudes are Weyl spinors $\psi^{\alpha}$ and $\bar{\psi}_{\dot{\alpha}}$, transforming as $(1 / 2,0)$ (undotted indices) and $(0,1 / 2)$ (dotted indices) representations of $S U(2) \times S U(2) \simeq S O(3,1)$, and Lorentz vectors $A_{\mu} \sigma_{\alpha \dot{\alpha}}^{\mu}$, transforming as $(1 / 2,1 / 2) .{ }^{2}$ In this language, the field strength is written as

$$
F_{\mu \nu} \sigma_{\alpha \dot{\alpha}}^{\mu} \sigma_{\beta \dot{\beta}}^{\nu} \equiv F_{\alpha \beta} \bar{\epsilon}_{\dot{\alpha} \dot{\beta}}+\bar{F}_{\dot{\alpha} \dot{\beta}} \epsilon_{\alpha \beta}
$$

in terms of its self-dual and antiself-dual parts $F$ and $\bar{F}$ [transforming respectively as $(1,0)$ and $(0,1)$ representations].

Our analysis is in terms of complex momenta $p \in \mathbb{C}$ : this allows one to make sense of three-point amplitudes on shell, even though these vanish for massless states with real kinematics. We need three well-known results, which we summarize here and discuss in the appendixes; see Refs. [9-11]. These are the following:

(1) Consider an amplitude $A_{n}$ with $n$ external legs (n-point amplitude), and let $A_{m}$ and $A_{m^{\prime}}$, with $m+m^{\prime}-2=n$, be two subamplitudes; see Fig. 1 . We define the net helicity of an on-shell amplitude, $h(A)$, as the sum of the helicities of all its external states, where all momenta are taken to be outgoing. Then one has

\footnotetext{
${ }^{2}$ We do not distinguish between fermions and antifermions except where explicitly mentioned, as this distinction is not crucial to our analysis. We denote a Weyl fermion or antifermion of helicity $+(-)$ with $\psi^{+}\left(\psi^{-}\right)$. When indicating a scattering amplitude, the symbol $\psi$ stands for either $\psi^{+}$or $\psi^{-}$.
}

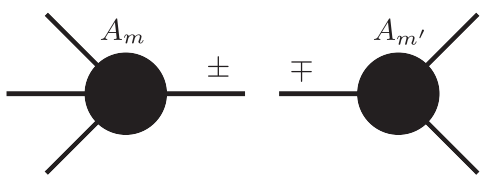

FIG. 1. When the factorization channel goes on shell, it propagates a well-defined helicity eigenstate and Eq. (3) holds.

$$
h\left(A_{n}\right)=h\left(A_{m}\right)+h\left(A_{m^{\prime}}\right)
$$

for all possible nonvanishing on-shell subamplitudes $A_{m}$ and $A_{m^{\prime}}$ that can be connected by a propagator. When the intermediate line goes on shell, the propagator generates a pole and the amplitude factorizes into the product of the two subamplitudes.

(2) Dimensional analysis, Little group scaling, and the three-particle special kinematics fix completely the form of the three-point amplitudes, and in particular relate their total helicity $h\left(A_{3}\right)$ to the dimensionality of the coupling $g$ characterizing the three-point vertex,

$$
\left|h\left(A_{3}\right)\right|=1-[g] .
$$

For instance, the triple gauge interaction of the SM is characterized by a dimensionless coupling, and the corresponding three-point on-shell amplitude has $|h|=1$. The $D=6$ operator $\mathcal{O}_{3 W}=\operatorname{tr}\left(W_{\mu \nu} W_{\rho}^{\nu} W^{\rho \mu}\right)$ instead appears in Eq. (1) with a coefficient $c_{3 W}$ with dimension $\left[c_{3 W}\right]=-2$, and thus generates a threepoint amplitude with $|h|=3$.

(3) Helicity selection rules in the SM force the following four-point amplitudes with $|h|=2$ to vanish:

$$
\begin{aligned}
A\left(V^{+} V^{+} V^{+} V^{-}\right) & =A\left(V^{+} V^{+} \psi^{+} \psi^{-}\right) \\
& =A\left(V^{+} V^{+} \phi \phi\right) \\
& =A\left(V^{+} \psi^{+} \psi^{+} \phi\right)=0 .
\end{aligned}
$$

These relations can be proved by means of the supersymmetric Ward identities (SWI) [12,13], as sketched in Appendix B. In the limit in which all uptype or all down-type Yukawa couplings vanish, the SM Lagrangian can be uplifted to a supersymmetric one, for which SWI hold. Such a theory has in addition an $R$-parity implying that the supersymmetric partners do not contribute at tree level to scattering amplitudes with external SM legs only. As a consequence, Eq. (5) holds for the SM.

\section{A. The standard model}

Within the SM, it follows from property 2 that threepoint vertices associated with marginal couplings have

$$
h\left(A_{3}^{\mathrm{SM}}\right)= \pm 1 .
$$

The three-scalar vertex (which would have vanishing total helicity) is absent in the SM in the massless limit (i.e. in the 
limit of unbroken EW symmetry). With the exception of the quartic scalar vertex, which has trivially total helicity $h\left(A_{4}\right)=0$, all four-point on-shell vertices in the SM can be made to vanish with a suitable definition of polarization vectors (this is a direct consequence of gauge invariance; see for example [10]). Therefore, all amplitudes with $n \geq 4$ have at least one pole, with the exception of $\phi^{4}$ whose helicity $h=0$ is known. Properties 1 and 3 then imply that all four-point amplitudes with fermions or gauge fields have vanishing total helicity, unless they involve both upand down-type Yukawa interactions $[9,14,15]$. The only exceptions are in fact the amplitudes $\psi^{+} \psi^{+} \psi^{+} \psi^{+}$and $\psi^{-} \psi^{-} \psi^{-} \psi^{-}$, which receive a contribution (proportional to the product of up- and down-type Yukawas) from the Higgs exchange and have $\left|h\left(A_{4}\right)\right|=2$. These known results are summarized in Table I.

\section{B. Beyond the standard model}

Local operators entering at a given order in the $1 / \Lambda$ expansion of an EFT can be redefined by making use of the equations of motion (EoM) derived at lower order. For example, it is always possible to rewrite $D=6$ operators by using the EoM of the renormalizable $D=4$ Lagrangian; the new effective Lagrangian differs from the original one by $D=8$ terms. This freedom allows one to systematically replace operators with more derivatives in terms of operators involving more fields. At the $D=6$ level, this procedure leads to the so-called Warsaw basis of operators introduced in Ref. [16]. This basis is particularly convenient to study $2 \rightarrow 2$ scattering processes for a number of reasons. First, it does not lead to corrections to two-point functions, which would modify the pole structure of propagators and thus require further discussion in relation with condition 1. Second, in this basis the relation between operators in the Lagrangian and scattering amplitudes is more transparent. Indeed, higher derivative operators proportional to the SM EoM generate effective vertices which vanish on shell, and thus give contributions to scattering amplitudes that do not factorize as in Eq. (3). As an example, consider the operator $\mathcal{O}_{B}=(i / 2) H^{\dagger} \stackrel{\leftrightarrow}{D^{\mu}} H \partial^{\nu} B_{\mu \nu}$, which appears in the SILH basis of Ref. [17]. It gives a vanishing contribution to the on-shell $H H B$ vertex (even for complex momenta), but contributes off shell to processes like $H H \rightarrow H H$ or $H H \rightarrow \psi \psi$. Indeed, by using the EoM it can be eliminated in favor of operators of the form $D^{2} H^{4}$ or $H^{2} D \psi^{2}$ that contribute to the previous processes via contact interactions. The Warsaw basis [16] only includes operators of the latter kind that factorize as described by condition 1 .

To make more explicit the relation between an operator $\mathcal{O}$ and the amplitudes it generates, it is useful to introduce the holomorphic and antiholomorphic weights of $\mathcal{O}$, as defined by Ref. [18]. For an arbitrary on-shell amplitude $A$ with $n(A)$ legs and helicity $h(A)$,

$$
w(A)=n(A)-h(A), \quad \bar{w}(A)=n(A)+h(A) .
$$

The weights of the operator $\mathcal{O}$ are then obtained by minimizing over all the amplitudes involving $\mathcal{O}$,

$$
w(\mathcal{O})=\min _{A}\{w(A)\}, \quad \bar{w}(\mathcal{O})=\min _{A}\{\bar{w}(A)\} .
$$

The point is that, as a consequence of Eq. (3) and the fact that $h\left(A_{3}^{\mathrm{SM}}\right)= \pm 1$ (from condition 2), building amplitudes with more SM interactions cannot decrease $w(A)$ and $\bar{w}(A)$, so that the weight is always determined by the amplitude with the smallest number of SM vertices. Since weights are defined in terms of on-shell amplitudes, they offer various advantages. First of all, they are gauge-invariant quantities characterizing with a unique label also operators, involving covariant derivatives or non-Abelian field strengths, that give contact contributions to different processes with different numbers of legs and total helicities: these contributions are in fact related by gauge invariance to amplitudes with factorization channels and therefore their weights are defined by the amplitude with the smallest number of legs, as described above. Moreover, they are well defined even for operators whose contribution to a given amplitude vanishes on shell (as for $\mathcal{O}_{B}$ discussed above). Finally, one can easily deduce from Eqs. (7) and (8) that the helicity of $n$-point amplitudes with one $\mathcal{O}$ insertion is constrained to be in the range

$$
\bar{w}(\mathcal{O})-n \leq h\left(A_{n}^{\mathcal{O}}\right) \leq n-w(\mathcal{O}) .
$$

Using these ingredients we can constrain the total helicity of $\mathrm{BSM}_{6}$ contributions to $2 \rightarrow 2$ scattering amplitudes. Let us start with the unique trivalent $F^{3}$ and $\bar{F}^{3}$ structures of $\mathcal{O}_{3 W}$ and $\mathcal{O}_{3 \tilde{W}}=\operatorname{tr}\left(W_{\mu \nu} W_{\rho}^{\nu} \tilde{W}^{\rho \mu}\right)$. Given the dimensionality of their coefficients, $\left[c_{i}\right]=-2$, Eq. (4) fixes the helicity of their contribution to the three-point amplitude up to a sign: $\left|h\left(A_{3}\right)\right|=3$. It is in fact not difficult to show that $F_{\alpha \beta}$ and $\bar{F}_{\dot{\alpha} \dot{\beta}}$ generate states with helicity +1 and -1 respectively (see $[19,20]$ ), which implies that $h\left(A_{3}\right)=$ +3 for $F^{3}$ and $h\left(A_{3}\right)=-3$ for $\bar{F}^{3}$. From Eq. (8) it follows that the weights of $F^{3}$ and $\bar{F}^{3}$ are respectively $(w, \bar{w})=$ $(0,6)$ and $(w, \bar{w})=(6,0)$. Equation (9) thus constrains the helicity of a four-point function with one insertion of either of these operators to be in the range $2 \leq\left|h\left(A_{4}\right)\right| \leq 4$ [more precisely, $2 \leq h\left(A_{4}\right) \leq 4$ for $F^{3}$ and $-4 \leq h\left(A_{4}\right) \leq-2$ for $\left.\bar{F}^{3}\right]$. Considering that $h\left(A_{4}\right)=0$ in the SM (for the amplitudes under consideration), this shows that no SMBSM interference is possible in this case. It is useful to directly verify the constraint of Eq. (9) for some specific amplitudes. Starting from a three-point amplitude with one $F^{3}$ insertion, for example, a four-point one is obtained by adding a SM cubic vertex [which has $h\left(A_{3}^{\mathrm{SM}}\right)= \pm 1$ as shown previously]. Then Eq. (3) implies that the four-point amplitude with only vectors, $V V V V$, has $|h|=4,2[19,21]$ 
TABLE II. Weights $(w, \bar{w})$ of the dimension-6 operators $\mathcal{O}_{i}$ in the Warsaw basis. Also shown are the number of legs $n_{\min }$ and corresponding helicity $h_{\min }$ of the smallest amplitude to which the operator contributes, and the naive estimate of its coefficient $c_{i}$. Operators with $\psi_{\alpha} \leftrightarrow \bar{\psi}_{\dot{\alpha}}$ and $F_{\alpha \beta} \leftrightarrow \bar{F}_{\dot{\alpha} \dot{\beta}}$ have $h_{i} \rightarrow-h_{i}$; hence $(w, \bar{w}) \leftrightarrow(\bar{w}, w)$.

\begin{tabular}{lcccc}
\hline \hline $\mathcal{O}_{i}$ & $n_{\min }$ & $h_{\min }$ & $(w, \bar{w})$ & $c_{i}$ \\
\hline$F^{3}$ & 3 & 3 & $(0,6)$ & $g_{*} / \Lambda^{2}$ \\
$F^{2} \phi^{2}, F \psi^{2} \phi, \psi^{4}$ & 4 & 2 & $(2,6)$ & $g_{*}^{2} / \Lambda^{2}$ \\
$\psi^{2} \bar{\psi}^{2}, \psi \bar{\psi} \phi^{2} D, \phi^{4} D^{2}$ & 4 & 0 & $(4,4)$ & $g_{*}^{2} / \Lambda^{2}$ \\
$\psi^{2} \phi^{3}$ & 5 & 1 & $(4,6)$ & $g_{*}^{3} / \Lambda^{2}$ \\
$\phi^{6}$ & 6 & 0 & $(6,6)$ & $g_{*}^{4} / \Lambda^{2}$ \\
\hline \hline
\end{tabular}

(notice that $F^{3}$ is not supersymmetrizable and condition 3 does not hold [11,22]). Similarly, the helicity of an amplitude $V V \psi \gamma \psi$ is $|h|=2$. Both results agree with the bound of Eq. (9).

Apart from $F^{3}$ and $\bar{F}^{3}$, the remaining $D=6$ operators of the Warsaw basis do not contribute to three-point amplitudes in the massless (high-energy) limit. Those contributing to four-point amplitudes are listed in the second and third row of Table II. The helicity of the four-point amplitudes in this case can be directly determined from the corresponding operators by noticing that $F_{\alpha \beta}\left(\bar{F}_{\dot{\alpha} \dot{\beta}}\right)$ creates states with helicity $+1(-1), \psi_{\alpha}\left(\psi_{\dot{\alpha}}\right)$ creates fermions or antifermions with helicity $+1 / 2(-1 / 2)$, and the helicity of scalars trivially vanishes. For example, an operator $F \psi^{2} \phi$ can excite states with net helicity $h_{\min }=+2$, which equals the helicity of the corresponding four-point amplitude. The results are reported in Table II: the operators in the second row lead to four-point amplitudes with helicity $\left|h\left(A_{4}\right)\right|=2$, and thus do not interfere with the SM. The operators in the third row give $\left|h\left(A_{4}\right)\right|=0$ and can thus interfere with the SM, but the corresponding amplitudes do not involve transversely polarized vector bosons. These results directly imply those of Table I.

In addition to the helicity selection rules derived above, $2 \rightarrow 2$ tree-level scattering amplitudes are constrained by additional selection rules in the massless limit. In particular, a simple one follows from weak isospin conservation. In the Warsaw basis, the only BSM contribution to the amplitudes $V V \psi \psi, V V V V$ comes from $F^{3}, \bar{F}^{3}$, and it can always be written as the product of two three-point amplitudes with a vector propagator $(V V \psi \psi \psi$ receives no quartic contribution from $D=6$ operators, while the quartic $V V V V$ vertex can always be made to vanish through a suitable choice of polarization vectors). The propagation of a vector boson implies a well-defined $S U(2)_{L}$ isospin structure of the external states produced at each vertex: they transform in the $\mathbf{3} \in \mathbf{3} \otimes \mathbf{3}$, which is totally antisymmetric and thus does not include pairs of identical bosons. For this reason amplitudes like ZZZZ, $\gamma \gamma \gamma \gamma, \psi \psi \psi Z Z$ and $\psi \psi \gamma \gamma \gamma$ can only be generated by $D=8$ operators. It is worth mentioning another selection rule which characterizes the $D=6$ effective theory in the massless limit. Its Lagrangian is invariant under the $Z_{2}$ chiral symmetry

$$
\phi \rightarrow-\phi, \quad \psi_{L} \rightarrow-\psi_{L}, \quad \psi_{R} \rightarrow+\psi_{R},
$$

as a direct consequence of $S U(2)_{L}$ invariance and of the SM quantum numbers [it is not possible to form operators which are singlets of $S U(2)_{L}$ with an odd number of $\psi_{L}$ and $H$ fields]. It follows that the amplitudes $V V V \phi$ and $V \phi \phi \phi$ identically vanish (in the massless limit), while the helicities of the fermion and antifermion in $V \psi \gamma \psi \phi$ are forced to be the same. Notice that these same conclusions are also a consequence of the helicity selection rules, since by the arguments presented above no four-point amplitude has total helicity $|h|=1,3$ in the SM or at the $D=6$ level. Using Eq. (10) might still be useful, however, as a quicker way to determine if a given amplitude vanishes, independently of helicity arguments.

To summarize, we have shown that working at tree level and in the massless (i.e. high-energy) limit, the BSM contribution never interferes with the SM one in $2 \rightarrow 2$ scattering amplitudes involving at least one transversely polarized vector boson. Interference is possible, instead, for amplitudes involving only scalars (including longitudinally polarized vector bosons) and fermions, such as $\psi \psi \rightarrow \psi \psi \psi$, $\psi \psi \rightarrow \phi \phi$ and $\phi \phi \rightarrow \phi \phi$. We comment on the practical implications of these results in Sec. III, but first we discuss how our analysis generalizes to the massive case and to higher-point scattering amplitudes.

\section{Higher-point amplitudes}

The helicity of amplitudes with five or more external legs can be easily determined in the SM by starting from that of four-point amplitudes, given in Table I, using the addition rule of Eq. (3) and knowing that three-point vertices change helicity by \pm 1 unit [Eq. (6)]. We find ${ }^{3}$

$$
\begin{gathered}
\left|h\left(A_{n \geq 5}^{\mathrm{SM}}\right)\right| \leq n-4 \\
\text { with } h \text { even (odd) for } n \text { even (odd). }
\end{gathered}
$$

For example, one has $h\left(A_{5}^{\mathrm{SM}}\right)= \pm 1, h\left(A_{6}^{\mathrm{SM}}\right)=0, \pm 2$ respectively for five-point and six-point SM amplitudes. By making use of the helicity selection rules for four-point amplitudes Eq. (5), combined with Eq. (3), one reproduces the well-known result that the first nonvanishing amplitudes with largest total helicity are the maximal helicity violating ones $[9,10]$.

\footnotetext{
${ }^{3}$ Notice that $n \geq 5$ amplitudes constructed from four-fermion subamplitudes with $|h|=2$ do not violate Eq. (11) as long as no cubic scalar interaction exists in the theory.
} 
The helicity of $\mathrm{BSM}_{6}$ amplitudes, including those with five or more external legs, can be constrained by using Eq. (9) and the weights reported in Table II. We find

$$
h_{\min }^{\mathcal{O}} \leq h\left(A_{n \geq n_{\min }}^{\mathcal{O}}\right) \leq h_{\max }^{\mathcal{O}}
$$

with $h$ even (odd) for $n$ even (odd)

\begin{tabular}{lcc}
\hline $\mathcal{O}_{i}$ & $h_{\min }^{\mathcal{O}}$ & $h_{\max }^{\mathcal{O}}$ \\
\hline$F^{3}$ & $6-n$ & $n$ \\
$F^{2} \phi^{2}, F \psi^{2} \phi, \psi^{4}$ & $6-n$ & $n-2$ \\
$\psi^{2} \bar{\psi}^{2}, \psi \bar{\psi} \phi^{2} D, \phi^{4} D^{2}$ & 0 & $n-4$ \\
$\psi^{2} \phi^{3}$ & $6-n$ & $n-4$ \\
$\phi^{6}$ & 0 & $n-6$ \\
\hline
\end{tabular}

where $n_{\min }$ is given in Table II for the various operators. The helicity of amplitudes with one insertion of conjugate operators (i.e. those with $F \rightarrow \bar{F}, \psi \rightarrow \bar{\psi}$ ) is obtained by exchanging $h_{\min } \leftrightarrow-h_{\max }$.

Notice that, both for the SM and $\mathrm{BSM}_{6}$, the total helicity is even (odd) if $n$ is even (odd). This follows from Little group scaling and the even dimensionality of the coupling constants $\left([g]=0\right.$ in the SM and $\left[c_{i}\right]=-2$ in $\left.\mathrm{BSM}_{6}\right)$; see Appendix A. In this respect [as well as to derive Eq. (11)], it is crucial that no scalar cubic vertex is present in the SM in the limit of unbroken EW symmetry. This selection rule automatically implies that amplitudes such as $V V V \phi$ or $V V \phi \phi \phi$ must vanish since they would have necessarily a total helicity with the wrong parity (a similar conclusion follows from chiral invariance, as seen above).

From Eq. (12) it follows that all $D=6$ operators contribute to amplitudes with $h\left(A_{5}\right)= \pm 1$ or $h\left(A_{6}\right)=0, \pm 2$ (the operator $\phi^{6}$ contributes only to six-point amplitudes), and can thus potentially interfere with the SM. Having the same total helicity is in fact a necessary but not sufficient condition for the SM and BSM amplitudes to have interference. The same net helicity can indeed be distributed differently on the external legs, in which case no interference occurs. Moreover, even in the case in which both the SM and $\mathrm{BSM}_{6}$ amplitudes have the same external helicities, additional selection rules can forbid the interference. As an example, consider the amplitude $g^{+} g^{+} \psi^{-} \psi^{-} \phi$ (where $\psi$ could be a top quark, $\phi$ a Higgs boson and the corresponding physical scattering $g g \rightarrow t \bar{t} H$ ), which is nonvanishing in the $\mathrm{SM}$ in the massless limit. The operator $\mathcal{O}_{3 \tilde{G}}$ contributes to the same helicity amplitude, but no interference occurs because $\mathcal{O}_{3 \tilde{G}}$ is $C P$ odd. Except for these particular cases, however, $D=6$ operators in general interfere with the SM at the level of $n \geq 5$ amplitudes. Noninterference seems therefore a peculiarity of four-point amplitudes.

\section{Finite-mass effects and radiative corrections}

The noninterference between $\mathrm{SM}$ and $\mathrm{BSM}_{6}$ amplitudes holds for $2 \rightarrow 2$ scatterings in the massless limit and at tree level. There are two main subleading effects which correct this result in real scattering processes: finite-mass corrections and radiative effects (one-loop corrections and real emissions).

Finite-mass effects have been extensively studied in the literature (see [23-27]) and can be easily included in our analysis. They can be parametrized in terms of

$$
\varepsilon_{V} \equiv \frac{m_{V}}{E}, \quad \varepsilon_{\psi} \equiv \frac{m_{\psi}}{E},
$$

where $m_{V(\psi)}$ is the vector (fermion) mass and $E$ the energy. In this article we are interested, in particular, to determine at which order in $\varepsilon_{V, \psi}$ the leading correction to a given amplitude appears. To this aim, the most effective procedure is to consider higher-point amplitudes with additional Higgs bosons in the external legs. Restricting to the phase space region where no momentum is transferred through a Higgs line corresponds in fact to setting the Higgs field to its vacuum expectation value (vev). One can thus identify the contribution to a given amplitude at order $k$ in the $\varepsilon$ expansion by considering a higher-point amplitude with $k$ insertions of the Higgs boson. Since the gauge and Yukawa interactions of the Higgs boson violate helicity by \pm 1 unit, this procedure allows one to easily determine the leading contribution to the $n=4$ amplitudes that are vanishing in the massless limit. For example, a transversely polarized vector can be turned into a longitudinal one (or vice versa) at order $\varepsilon_{V}$ through the insertion of a vertex $\phi^{*} \partial_{\mu} \phi A^{\mu}$, by setting $\phi^{*}$ to its vev. This follows from the equivalence theorem $[28,29]$, which states that, at leading order in the $\varepsilon_{V}$ expansion, a longitudinal polarization can be replaced by the corresponding wouldbe Nambu-Goldstone boson. For fermions, the Yukawa interaction $\psi^{\alpha} \psi_{\alpha} \phi\left(\psi_{\dot{\alpha}} \psi^{\dot{\alpha}} \phi\right)$ has total helicity $h=+1(-1)$ and its insertion leads to a flip of the fermion chirality at order $\varepsilon_{\psi}$, according to Eq. (3).

In general, the ${\mathrm{SM}-\mathrm{BSM}_{6}}_{6}$ interference in an $n=4$ amplitude arises at order $k_{\mathrm{SM}}+k_{\mathrm{BSM}}$ in $\varepsilon$ if nonvanishing $\mathrm{SM}$ and $\mathrm{BSM}_{6}$ amplitudes exist with respectively $k_{\mathrm{SM}}$ and $k_{\mathrm{BSM}}$ additional external Higgs fields. The power $k_{\mathrm{SM}}+$ $k_{\mathrm{BSM}}$ is always even, as a consequence of the fact that the total helicity of an $n$-point amplitude is even (odd) if $n$ is even (odd). Hence, the interference in $n=4$ scattering amplitudes with one or more transversely polarized vector bosons is suppressed at least by two powers of $\varepsilon$. As an example of the case $k_{\mathrm{SM}}=0, k_{\mathrm{BSM}}=2$ consider the amplitude $V^{+} V^{+} V^{-} V^{-}$: it is nonzero in the SM but its $\mathrm{BSM}_{6}$ contribution vanishes in the massless limit. The sixpoint amplitude $V^{+} V^{+} V^{-} V^{-} \phi \phi$, on the other hand, is generated by $F^{3}$ and $F^{2} \phi^{2}$. At order $\varepsilon_{V}^{2}$ this leads to a contribution to the $n=4$ amplitude and thus to an interference with the SM. As a second example, the amplitude $V^{+} V^{+} V^{+} V^{+}$has interference at order $\varepsilon_{V}^{4}$ for $k_{\mathrm{SM}}=4, k_{\mathrm{BSM}}=0$. The $\mathrm{SM}$ contribution arises from $V^{+} V^{+} V^{+} V^{+} \phi \phi \phi \phi$ after taking four Higgs vevs, while the BSM one is generated already in the massless limit from 
insertions of $F^{3}$. Finally, an example with $k_{\mathrm{SM}}=1$, $k_{\mathrm{BSM}}=1$ is given by the amplitude $V^{+} \psi^{+} \psi^{-} \phi$, which vanishes in the massless limit both in the SM and beyond. The five-point amplitude $V^{+} \psi^{+} \psi^{-} \phi \phi$, on the other hand, is generated through one insertion of any of the operators $F^{3}$, $F^{2} \phi^{2}, F \psi^{2} \phi$ and $\psi \bar{\psi} \phi^{2} D$, and is nonvanishing also in the SM. The interference arises at order $\varepsilon_{V}^{2}$ (in the case of $F^{3}$, $F^{2} \phi^{2}$ and $F \psi^{2} \phi$ ) and $\varepsilon_{V} \varepsilon_{\psi}$ (for $\psi \bar{\psi} \phi^{2} D$ ).

Contributions from fermion mass insertions are in general subdominant compared to those from vector mass insertions, with the exception of processes involving the top quark, for which $\varepsilon_{\psi} \approx \varepsilon_{V}$, like $b W \rightarrow t h$ [30] and $t W \rightarrow t W$ [31]. In processes involving gluons instead of EW vector bosons, top-quark mass insertions are in fact the only way to get interference between $\mathrm{SM}$ and $\mathrm{BSM}_{6}$. An example is given by the scattering $g g \rightarrow t \bar{t}$ [32-34], where the operators $F^{3}$ and $F \psi^{2} \phi$ (where $F$ is a gluonic field strength) interfere at order $\varepsilon_{\psi}^{2}$ with the SM.

We summarize our results for processes involving EW vector bosons in Table III, where we report the order in $\varepsilon_{V}$ at which a given helicity amplitude appears, in the SM and $\mathrm{BSM}_{6}$. For simplicity we work in the limit of vanishing Yukawa couplings and do not specify which $D=6$ operators give rise to an interference. In all cases the interference term in the amplitude squared goes like a constant in the highenergy limit, $E \gg m_{W}$, except for the processes in the first line of the two panels, where it scales as $1 / E^{2}$.

TABLE III. Leading power of $\varepsilon_{V}$ at which a given helicity amplitude is generated in the SM and $\mathrm{BSM}_{6}$. The first column indicates the process and the polarizations of the external states: 0 corresponds to a longitudinally polarized vector boson or to a Higgs boson, \pm to a transversely polarized vector boson $V=W$, $Z, \gamma$, and $\pm \frac{1}{2}$ to a fermion. Yukawa couplings have been neglected for simplicity, and only nonvanishing amplitudes are shown. Conjugate amplitudes with $+\leftrightarrow-$ follow the same pattern. The $\varepsilon_{V}^{0}$ entries match those of Table I.

\begin{tabular}{lcc}
\hline \hline Channel & $\mathrm{SM}$ & $\mathrm{BSM}_{6}$ \\
\hline++++ & $\varepsilon_{V}^{4}$ & $\varepsilon_{V}^{0}$ \\
+++- & $\varepsilon_{V}^{2}$ & $\varepsilon_{V}^{0}$ \\
++-- & $\varepsilon_{V}^{0}$ & $\varepsilon_{V}^{2}$ \\
$+\frac{1}{2}-\frac{1}{2}++$ & $\varepsilon_{V}^{2}$ & $\varepsilon_{V}^{0}$ \\
$+\frac{1}{2}-\frac{1}{2}+-$ & $\varepsilon_{V}^{0}$ & $\varepsilon_{V}^{2}$ \\
$+\frac{1}{2}-\frac{1}{2} 0+$ & $\varepsilon_{V}^{1}$ & $\varepsilon_{V}^{1}$ \\
$+\frac{1}{2}-\frac{1}{2} 00$ & $\varepsilon_{V}^{0}$ & $\varepsilon_{V}^{0}$ \\
\hline \hline Channel & & \\
\hline $0+++$ & $\mathrm{SM}$ & $\mathrm{BSM}_{6}$ \\
$0++-$ & $\varepsilon_{V}^{3}$ & $\varepsilon_{V}^{1}$ \\
$00++$ & $\varepsilon_{V}^{1}$ & $\varepsilon_{V}^{1}$ \\
$00+-$ & $\varepsilon_{V}^{2}$ & $\varepsilon_{V}^{0}$ \\
$000+$ & $\varepsilon_{V}^{0}$ & $\varepsilon_{V}^{2}$ \\
0000 & $\varepsilon_{V}^{1}$ & $\varepsilon_{V}^{1}$ \\
\hline \hline
\end{tabular}

Let us now consider radiative corrections. In general, oneloop corrections to four-point amplitudes violate the helicity selection rules discussed in Secs. II A and II B, and thus generate a nonvanishing interference. The most relevant contribution arises from QCD corrections to amplitudes with external quarks or gluons. Pure EW loop corrections have a similar effect but are numerically smaller. The emission of an extra gluon transforms a four-point to a five-point amplitude and can also lead to interference. It is well known (see [9]) that in the limit in which one parton becomes soft, an $n$-point color-ordered amplitude factorizes into the product of the $(n-1)$-point amplitude made of the remaining hard partons times a singular eikonal factor. Because of the helicity selection rules controlling four-point amplitudes, it is thus clear that soft emissions of extra gluons or quarks cannot lead to interference; that is, the interference vanishes in the soft limit. Similarly, when two partons $i$ and $j$ become collinear, an $n$-point colorordered amplitude factorizes into the $(n-1)$-point amplitude obtained by replacing the $i j$ pair with an effective parton carrying its momentum, times a singular splitting function (see [9]). Starting with a five-point amplitude and taking the collinear limit, the selection rules acting on fourpoint amplitudes force the helicity of the effective parton to be opposite in the $\mathrm{SM}$ and $\mathrm{BSM}_{6}$ cases. This implies that the $\mathrm{SM}_{-}-\mathrm{BSM}_{6}$ interference term, once integrated over the full phase space, is nonsingular; that is, the collinear singularity of the amplitude squared vanishes in the total cross section at order $1 / \Lambda^{2}$ [21]. The absence of soft and collinear singularities in real emission processes in turn implies that $\mathrm{SM}$ and $\mathrm{BSM}_{6}$ amplitudes which vanish at tree level are IR finite at one loop [21]. Hence, although oneloop QCD corrections and real emissions of extra gluons do lead to interference between $\mathrm{SM}$ and $\mathrm{BSM}_{6}$ amplitudes, no logarithmic enhancement is present in the collinear and soft limits. This means that the interference is suppressed by a factor $\alpha_{s} / 4 \pi$, where $\alpha_{s}$ is evaluated at the high-energy scale characterizing the scattering process.

Summarizing, interference between SM and $\mathrm{BSM}_{6}$ can arise in $2 \rightarrow 2$ exclusive processes as a result of one-loop corrections and finite-mass effects, with a relative suppression of order, respectively, $\alpha_{s} / 4 \pi$ (or $\alpha_{\mathrm{em}} / 4 \pi$ for processes without colored particles) and $m_{W}^{2} / E^{2}$. Mass effects dominate at lower energies, while radiative corrections take over at energies $E \gtrsim m_{W} \sqrt{4 \pi / \alpha_{s}}$. Similar conclusions hold in the case of processes where the final state is defined inclusively with respect to the emission of additional QCD radiation. In this case the leading $\mathrm{SM}^{-\mathrm{BSM}_{6}}$ interference arises also from amplitudes with an additional gluon, while the pure SM contribution stems at lowest perturbative order.

A way to access the $1 / \Lambda^{2}$ corrections from $D=6$ operators without any relative suppression of the signal compared to the SM irreducible background is to instead consider exclusive $2 \rightarrow 3$ scattering processes, where the additional particle could be a hard gluon. In this case, as 
discussed in Sec. II C, the interference term arises at tree level also in the massless limit, so that both the SM and SM-BSM 6 interference are equally suppressed. This strategy was for example proposed by the authors of Ref. [21], who suggested constraining the operator $\mathcal{O}_{3 G}$ using three-jet events to avoid the noninterference of four-point amplitudes already noticed in [35]. For final states with one extra hard gluon, the gain in signal $/ \sqrt{\text { background }}$, compared to $2 \rightarrow 2$ loop processes, is only of order $\sqrt{4 \pi / \alpha_{s}}$.

\section{PHENOMENOLOGICAL IMPLICATIONS}

The helicity selection rules of Sec. II imply that the contributions from operators $F^{3}, F^{2} \phi^{2}, F \psi^{2} \phi$ and their conjugates never interfere with the SM ones in the $2 \rightarrow 2$ scattering process at high energy and tree level, and that the interference is suppressed by powers of $\left(m_{W} / E\right)^{2}$ or $\alpha_{s} / 4 \pi$. What does this imply about the phenomenology at colliders?

The impact of BSM precision searches performed using the EFT approach can be easily quantified in the context of theories characterized by a single microscopic scale $\Lambda$ and a single (new) coupling $g_{*}$ [17]. This provides a power counting prescription to estimate the size of the effective coefficients $c_{i}$ in Eq. (1) in terms of the parameters of the UV theory,

$$
c_{i}^{(D)} \sim \frac{g_{*}^{n_{i}-2}}{\Lambda^{D-4}},
$$

where $n_{i}$ counts the number of fields in $\mathcal{O}_{i}^{(D)}$. The corresponding estimate of the coefficient of each operator is reported in the last column of Table II. Such power counting smoothly interpolates between the strong coupling limit $g_{*} \sim 4 \pi$, where it is equivalent to naive dimensional analysis [36], and the weak coupling limit $g_{*} \lesssim g_{\mathrm{SM}}$. Additional symmetries and selection rules can lower the estimates of Eq. (14) $[1,2,17]$.

To appreciate the relevance of noninterference, let us first discuss the BSM amplitudes which do interfere with the SM, such as $A(\phi \phi \phi \phi)$ in the scattering of four longitudinally polarized vector bosons. This process receives a contribution from the operator $\mathcal{O}_{H}=(1 / 2)\left(\partial^{\mu}|H|^{2}\right)^{2}$, with estimated coefficient $c_{H} \approx g_{*}^{2} / \Lambda^{2}$, which may capture for example the effect of Higgs compositeness or the virtual exchange of heavy vectors coupled to the Higgs current with strength $g_{*}$. At the $D=8$ level, higher derivative operators also contribute with estimated coefficients $c^{(8)} \approx g_{*}^{2} / \Lambda^{4}$ (for example they may capture higher-order terms in the $p^{2} / \Lambda^{2}$ expansion of the propagator of the heavy vectors). The corresponding contributions to the $V_{L} V_{L} \rightarrow V_{L} V_{L}$ scattering cross section are, schematically,

$\sigma_{L} \sim \frac{g_{\mathrm{SM}}^{4}}{E^{2}}[1+\underbrace{\frac{g_{*}^{2}}{g_{\mathrm{SM}}^{2}} \frac{E^{2}}{\Lambda^{2}}}_{\mathrm{BSM}_{6} \times \mathrm{SM}}+\underbrace{\frac{g_{*}^{4}}{g_{\mathrm{SM}}^{4}} \frac{E^{4}}{\Lambda^{4}}}_{\mathrm{BSM}_{6}{ }^{2}}+\underbrace{\frac{g_{*}^{2}}{g_{\mathrm{SM}}^{2}} \frac{E^{4}}{\Lambda^{4}}}_{\mathrm{BSM}_{8} \times \mathrm{SM}}+\cdots]$.

Since $E \ll \Lambda$ for the EFT expansion to make sense, $D=8$ effects are always subdominant, while the $\mathrm{BSM}_{6}-\mathrm{SM}$ interference term always dominates for weakly coupled theories. In the case of strongly coupled theories, $g_{*}>g_{\mathrm{SM}}$, the BSM contribution is larger than the SM one at energies $E \gtrsim \Lambda\left(g_{\mathrm{SM}} / g_{*}\right)$, where the $\left(\mathrm{BSM}_{6}\right)^{2}$ term dominates. We illustrate this situation in the left panel of Fig. 2. Similar arguments hold for $\psi \psi \psi \rightarrow \psi \psi$ [37] and $\psi \psi \rightarrow \phi \phi ~[38]$.
LLLL

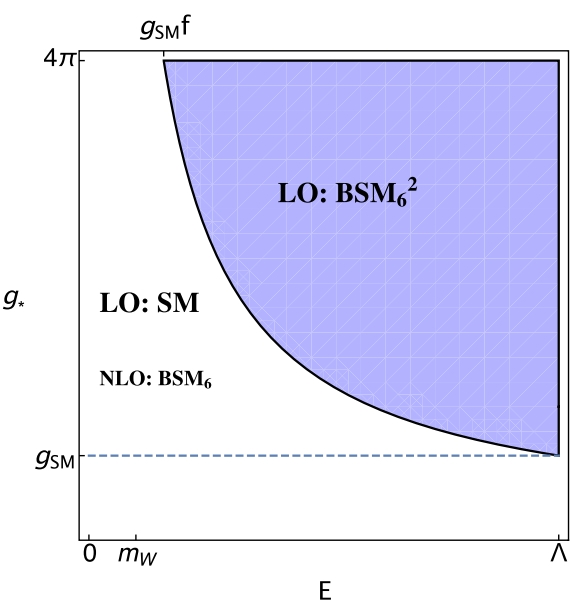

LLTT

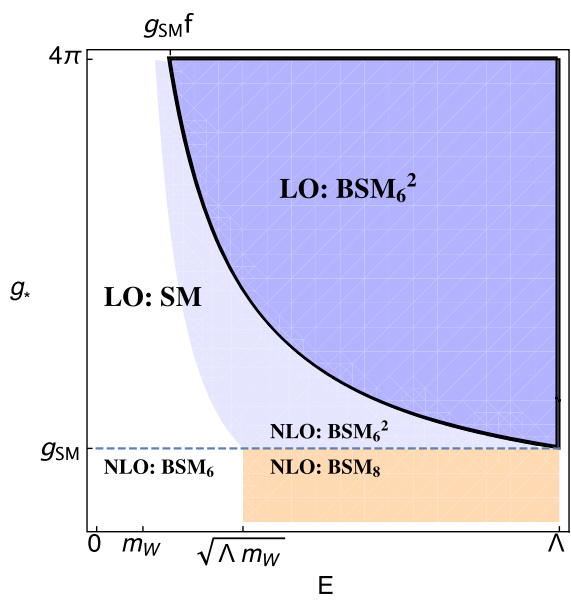

TTTT

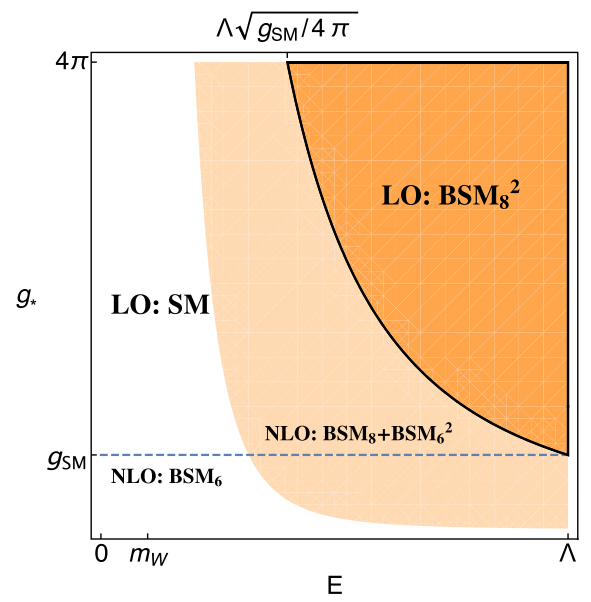

FIG. 2. A schematic representation of the relative size of different contributions to the $V V V V$ scattering cross sections, with polarization LLLL (left panel), LLTT (central panel) and TTTT (right panel). LO and NLO denote the leading and next-to-leading contributions to the cross section, respectively. In the white region the SM dominates and the leading BSM correction comes from the $\mathrm{BSM}_{6}-\mathrm{SM}$ interference (denoted as $\mathrm{BSM}_{6}$ ). BSM noninterference is responsible for the light-shaded blue and orange regions, where the BSM, although it is only a small perturbation around the $\mathrm{SM}$, is dominated by terms of order $E^{4} / \Lambda^{4}$, either from $\left(\mathrm{BSM}_{6}\right)^{2}$ or from the $\mathrm{BSM}_{8}-\mathrm{SM}$ interference (denoted as $\mathrm{BSM}_{8}$ ). 
As an example where the noninterference is at work, consider the scatterings $V_{T} V_{T} \rightarrow V_{L} V_{L}$ (with its crossings) and $V_{T} V_{T} \rightarrow V_{T} V_{T}$. We are inclusive on the transverse polarizations, implying a sum (or average) over them in the following discussion. We later highlight the advantages of an angular distribution analysis able to select the final-state polarizations. Let us discuss first the scattering $V_{T} V_{T} \rightarrow$ $V_{L} V_{L}$. In this case the largest BSM correction potentially comes from operators of the form $F^{2} \phi^{2}$ and $F^{3}$, whereas $\phi^{4} D^{2}$ and $\phi^{6}$ contribute only at subleading level in $\varepsilon_{V}$. The helicity selection rules of Sec. II imply that the interference with the SM is suppressed and arises at order $\varepsilon_{V}^{2}$ in the mass insertion or via one-loop EW corrections. It turns out that the latter effect is always subdominant in the following discussion and is thus neglected for simplicity. The naive estimate of the various terms entering the cross section is different, according to Eq. (14), for the operators $F^{2} \phi^{2}$ and $F^{3}$. Assuming that only $F^{2} \phi^{2}$ contributes, one finds, schematically,

$$
\begin{aligned}
\sigma_{L T} \sim & \frac{g_{\mathrm{SM}}^{4}}{E^{2}}[1+\overbrace{\frac{g_{*}^{2}}{g_{\mathrm{SM}}^{2}} \frac{m_{W}^{2}}{\Lambda^{2}}}^{\mathrm{BSM}_{6} \times \mathrm{SM}^{2}}+\overbrace{\frac{g_{*}^{4}}{g_{\mathrm{SM}}^{4}} \frac{E^{4}}{\Lambda^{4}}}^{\mathrm{BSM}_{6}{ }^{2}} \\
& +\underbrace{\frac{g_{*}^{2}}{g_{\mathrm{SM}}^{2}} \frac{E^{4}}{\Lambda^{4}}}_{\mathrm{BSM}_{8} \times \mathrm{SM}}+\cdots] .
\end{aligned}
$$

The importance of the various terms is illustrated in the central panel of Fig. 2. For small enough energy, where the BSM gives a small perturbation to the SM prediction, the $\mathrm{BSM}_{6}-\mathrm{SM}$ interference dominates. The suppression of the latter has however an important impact on the behavior at higher energies. If $g_{*}>g_{\mathrm{SM}}$, it implies a precocious onset of the regime where the $\left(\mathrm{BSM}_{6}\right)^{2}$ term must be included: for $\left(m_{W} \Lambda g / g_{*}\right)^{1 / 2}<E<\Lambda g / g_{*}$, corresponding to the light blue region of the figure, the SM still dominates but the $\left(\mathrm{BSM}_{6}\right)^{2}$ term gives the largest correction; for higher energies $\left(\mathrm{BSM}_{6}\right)^{2}$ eventually dominates the cross section. For weak or superweak UV completions, $g_{*}<g_{\mathrm{SM}}$, the largest correction to the SM prediction comes from $D=8$ operators, in particular from the interference $\mathrm{BSM}_{8}-\mathrm{SM}$, as soon as the energy is larger than $\sim \sqrt{m_{W} \Lambda}$ (light orange region in the figure). In this case, an EFT analysis in terms of $D=6$ operators alone is insufficient.

Yet a different energy behavior is found for the scattering $V_{T} V_{T} \rightarrow V_{T} V_{T}$, where $F^{3}$ gives the leading correction, while the operators $F^{2} \phi^{2}, \phi^{4} D^{2}$ and $\phi^{6}$ contribute at subleading order in $\varepsilon_{V}$. (Similar conclusions are in fact obtained also for $V_{T} V_{T} \rightarrow V_{L} V_{L}$ in the case in which only $F^{3}$ contributes.) Because the coefficient of $F^{3}$ scales with only one power of $g_{*}$ according to Eq. (14), the size of the $D=6$ terms [both $\left(\mathrm{BSM}_{6}\right)^{2}$ and the $\mathrm{BSM}_{6}-\mathrm{SM}$ interference] is suppressed compared to Eq. (16). The correction from $D=8$ operators might not carry a similar suppression, as it happens for example for the $F^{2} \bar{F}^{2}$ operator, whose coefficient has a naive estimate $c^{(8)} \approx g_{*}^{2} / \Lambda^{4}$. The different contributions to the cross section can thus be schematically summarized as follows:

$$
\begin{gathered}
\sigma_{T} \sim \frac{g_{\mathrm{SM}}^{4}}{E^{2}}[1+\overbrace{\frac{g_{*}}{g_{\mathrm{SM}}} \frac{m_{W}^{2}}{\Lambda^{2}}}^{\mathrm{BSM}_{6} \times \mathrm{SM}}+\overbrace{\frac{g_{*}^{2}}{g_{\mathrm{SM}}^{2}} \frac{E^{4}}{\Lambda^{4}}}^{\mathrm{BSM}_{6}{ }^{2}} \\
+\underbrace{g_{*}^{2}}_{\mathrm{BSM}_{8} \times \mathrm{SM}} \frac{E^{4}}{\Lambda^{4}}+\underbrace{\frac{g_{*}^{4}}{g_{\mathrm{SM}}^{4}} \frac{E^{8}}{\Lambda^{8}}}_{\mathrm{BSM}_{8}{ }^{2}}+\cdots] .
\end{gathered}
$$

Independently of the size of the interference term, this expression shows that as soon as the $D=6$ effects become bigger than the SM [for $E>\Lambda\left(g / g_{*}\right)^{1 / 2}$ ], the $D=8$ contribution takes over and dominates the cross section [1]. Noninterference implies a precocious onset of the regime where $D=8$ operators must be included: for energies $E>\sqrt{m_{W} \Lambda}\left(g / g_{*}\right)^{1 / 4}$ the dominant correction to the SM comes both from $\left(\mathrm{BSM}_{6}\right)^{2}$ and from the $\mathrm{BSM}_{8}-\mathrm{SM}$ interference. The situation is illustrated in the right panel of Fig. 2. We conclude that, for the scattering $V_{T} V_{T} \rightarrow V_{T} V_{T}$, inclusion of $D=8$ operators is crucial in a vast energy region above threshold.

So far we have considered processes where the transverse polarizations of the vector bosons are treated inclusively, i.e. they are summed over in the final state and averaged in the initial one. This in practice corresponded to a sum over two different kinds of helicity amplitudes in each process, one in which the SM arises at higher order in $\varepsilon_{V}$, the other in which it is the $\mathrm{BSM}_{6}$ amplitude to be suppressed. As an example, consider the amplitudes $A\left(\phi \phi V^{ \pm} V^{ \pm}\right)$and $A\left(\phi \phi V^{ \pm} V^{\mp}\right)$ which have both been included to obtain the estimate of the cross section of $V_{L} V_{L} \rightarrow V_{T} V_{T}$ Eq. (16). This suggests that an experimental analysis able to distinguish the polarizations in the final state could be used to select those processes where the SM amplitude arises at subleading order in the mass insertion (while the $\mathrm{BSM}_{6}$ contribution is unsuppressed). This would increase the significance of the signal compared to the irreducible SM background. Another example is the process $\psi \bar{\psi} \rightarrow V^{ \pm} V^{ \pm}$, relevant for the study of anomalous triple gauge couplings, where the polarizations of the final-state vector bosons are equal (while those of the fermions are averaged over). In this process the leading SM amplitude arises at order $\varepsilon_{V}$, while the $\mathrm{BSM}_{6}$ one is unsuppressed. Selecting the finalstate polarizations through an angular distribution analysis can thus improve the sensitivity on new physics. More in general, an exclusive approach to the final state can lead to a parametric enhancement of the signal 
significance compared to the naive estimates which follow from our analysis of Sec. II. An interesting example in this sense is given by the proposal of Ref. [21] to study three-jet events by exploiting the distribution of collinear jet pairs under azimuthal rotations as a way to (parametrically) enhance the sensitivity on the operator $\mathcal{O}_{3 G}$.

We conclude our discussion on the impact of noninterference by noticing an interesting fact: with the exception of $\psi ж ж \psi \psi$, the SM amplitudes that do interfere with the BSM are accidentally suppressed in their contribution to inclusive cross sections. Indeed, the contribution of the $V_{L} V_{L} \rightarrow V_{L} V_{L}$ amplitude to the $V V \rightarrow V V$ inclusive cross section is accidentally suppressed in the SM by a factor $\lesssim 1 / 500$ with respect to $V_{T} V_{T} \rightarrow V_{T} V_{T}$ [39]. Similarly, in the SM the contribution of $\psi \bar{\psi} \rightarrow V_{L} V_{L}$ is only $\sim 1 / 10$ of the $\psi \bar{\psi} \rightarrow V V$ total cross section [40]. Therefore, despite arising at leading order in the high-energy limit, the $\mathrm{SM}^{-\mathrm{BSM}_{6}}$ interference is anyway suppressed by the fact that the SM amplitude is small. Since at the LHC current experimental studies mostly focus on unpolarized cross sections, this implies an additional obstacle in extracting useful information on $D=6$ operators through their interference with the SM.

\section{CONCLUSIONS}

In this paper we have shown that in a theory where the SM is extended by $D=6$ effective operators, tree-level four-point amplitudes are subject to helicity selection rules in the massless limit. These forbid the interference between SM and $D=6$ BSM contributions for all amplitudes involving at least one transversely polarized vector boson. Such noninterference was noticed before in the literature for a few specific operators and processes (see $[21,32,35]$ ). Our analysis extends the result in a systematic way to all the $D=6$ operators, identifying the exceptions in which interference can instead arise. At the phenomenological level, our analysis implies that the BSM effects that are naively expected to be dominant in an EFT approach, i.e. those captured by the interference of $D=6$ effective operators with the SM, are suppressed in the high-energy limit. The interference only arises at next-to-leading order in an expansion in mass over energy and in the one-loop perturbative parameter $\alpha_{s} / 4 \pi$ (or $\alpha_{\mathrm{em}} / 4 \pi$ for processes not involving colored particles). Interestingly, some of the remaining amplitudes which do feature interference are accidentally small in the SM, implying anyway a small interference. This leads to a reduced sensitivity on new physics, especially in the case of analyses that are inclusive on the polarizations of the final-state particles. Furthermore, in many cases of interest and in particular when the underlying theory is weakly coupled, a generic EFT analysis in terms of $D=6$ operators alone is insufficient, as $D=8$ ones give an equally large (if not larger) contribution.

\section{ACKNOWLEDGMENTS}

We thank Clifford Cheung, Claude Duhr, Joan Elias Miro, Ricardo Monteiro, Yael Shadmi, Giovanni Villadoro and Giulia Zanderighi for interesting discussions. C. S. M. is supported by the São Paulo Research Foundation (FAPESP) under Grant No. 2012/21627-9. The work of R. C. was partly supported by the ERC Advanced Grant No. 267985 "Electroweak symmetry breaking, flavor and dark matter: One solution for three mysteries (DaMeSyFla)."

\section{APPENDIX A: THE SPINOR HELICITY FORMALISM}

We summarize here some useful results on the spinor helicity formalism (see Refs. [9,10] for a review). In this approach, the fundamental objects defining the scattering amplitudes are the spinors $|p\rangle^{\dot{a}}$ and $\left.\mid p\right]^{a}$ transforming as $(1 / 2,0)$ and $(0,1 / 2)$ under $S U(2) \times S U(2) \simeq S O(3,1)$. They are independent solutions of the massless Dirac equation,

$$
\begin{array}{ll}
\left.v_{+}(p)=(\mid p]_{a}, 0\right) & \bar{u}_{+}(p)=\left(\left[\left.p\right|^{a}, 0\right)\right. \\
v_{-}(p)=\left(0,|p\rangle^{\dot{a}}\right) & \bar{u}_{-}(p)=\left(0,\left\langle\left. p\right|_{\dot{a}}\right),\right.
\end{array}
$$

where the subscript \pm corresponds to an helicity $h= \pm 1 / 2$. Dotted and undotted indices are raised/lowered with the two-index Levi-Civita tensor. A $(1 / 2,1 / 2)$ Lorentz vector is written in terms of the spinors as $-\not p=|p\rangle[p|+| p]\langle p|$, while the polarization vectors for spin- 1 massless bosons are

$$
\epsilon_{-}^{\mu}(p ; q)=\frac{\left\langle p\left|\gamma^{\mu}\right| q\right]}{\sqrt{2}[q p]}, \quad \epsilon_{+}^{\mu}(p ; q)=\frac{\left\langle q\left|\gamma^{\mu}\right| p\right]}{\sqrt{2}\langle q p\rangle},
$$

where $q$ is a reference vector whose arbitrariness reflects gauge invariance. The products of angle and square spinors $\langle p q\rangle \equiv\left\langle\left. p\right|_{\dot{a}} \mid q\right\rangle^{\dot{a}}$ and $[p q] \equiv\left[\left.p\right|^{a} \mid q\right]_{a}$ satisfy the properties

$$
\langle p p\rangle=\langle p q]=0, \quad\langle p q\rangle[p q]=2 p \cdot q=(p+q)^{2}
$$

for any $p$ and $q$.

In many theories the basic building blocks for all scattering amplitudes are three-point amplitudes. Momentum conservation in the three-point vertex $\left(p_{1}^{\mu}+p_{2}^{\mu}+p_{3}^{\mu}\right)=0$ and the on-shell condition $p_{i}^{2}=0$ imply $p_{i} \cdot p_{j}=0$, which in bracket notation reads

$$
\langle 12\rangle[12]=0, \quad\langle 23\rangle[23]=0, \quad\langle 31\rangle[31]=0 .
$$

The only nontrivial solutions are $\langle 12\rangle=\langle 23\rangle=\langle 31\rangle=0$ or $[12]=[23]=[31]=0$. This means that the threeparticle amplitudes can depend only on square or angle brackets, never on both.

Spinors are defined up to a multiplicative factor, referred to as Little group scaling, 


$$
\left.\left.\left|p_{i}\right\rangle \rightarrow t_{i}\left|p_{i}\right\rangle \text { and } \mid p_{i}\right] \rightarrow t_{i}^{-1} \mid p_{i}\right],
$$

which leaves the momentum $\left.\left(p_{i}\right)_{a \dot{b}}=-\mid p_{i}\right]_{a}\left\langle\left. p_{i}\right|_{\dot{b}}\right.$ invariant. Under such transformation the polarization vector of a spin-1 particle scales as $t_{i}^{-2 h_{i}}$ if it has helicity $h_{i}= \pm 1$. An on-shell tree-level amplitude thus scales as $t^{-2 h_{i}}$ under the rescaling of a particle $i$ with helicity $h_{i}$, and as $t^{-2 h}$, with $h=\sum_{i} h_{i}$, when all particles are rescaled. We have seen that the special three-particle kinematics described below Eq. (A4) implies that a three-point amplitude must depend either on square or angle brackets. Little group scaling and the request of locality then fix completely the form of the amplitude to be (at tree level)

$$
A_{3}=g \begin{cases}\langle 12\rangle^{r_{3}}\langle 23\rangle^{r_{1}}\langle 31\rangle^{r_{2}} & \text { for } h\left(A_{3}\right) \leq 0 \\ {[12]^{\bar{r}_{3}}[23]^{\bar{r}_{1}}[31]^{\bar{r}_{2}}} & \text { for } h\left(A_{3}\right) \geq 0\end{cases}
$$

where $\quad r_{1}=h_{1}-h_{3}-h_{2}, \quad r_{2}=h_{2}-h_{1}-h_{3} \quad$ and $r_{3}=h_{3}-h_{2}-h_{1}$, while $\bar{r}_{i}=-r_{i}$. From simple dimensional analysis it follows that the total helicity of a three-point tree-level amplitude, $h\left(A_{3}\right)$, is fixed by the dimensionality of the coupling constant $g$; such a relation is given by Eq. (4) in the main text.

Similar arguments applied to $n$-point amplitudes imply that the total helicity $h\left(A_{n}\right)$ satisfies

$$
n-h\left(A_{n}\right)+[g]=\text { even }
$$

where $[g]$ is the sum of the dimensions of the couplings contributing to the amplitude. For $[g]$ even, in particular, it follows that $h\left(A_{n}\right)$ has the same parity as $n$.

\section{APPENDIX B: SUPERSYMMETRIC WARD IDENTITIES}

As long as all up-type or all down-type Yukawa couplings vanish, the SM fields and interactions can be embedded in a supersymmetric Lagrangian with $R$-parity. When both kinds are nonvanishing, however, holomorphy of the superpotential requires the introduction of an additional Higgs doublet or explicit supersymmetry breaking. Most SM tree-level amplitudes (all those not involving simultaneously up- and down-type Yukawas) can thus be written in supersymmetric form. $R$-parity implies that no supersymmetric state propagates in the internal lines, so that these amplitudes are effectively supersymmetric. This feature is generically lost in $\mathrm{BSM}_{6}$, although some operators can still be uplifted to a supersymmetric form [41].

Supersymmetry implies important relations between scattering amplitudes [12] (see [9,19] for a review). Since the supercharge $Q(\xi)=\bar{\xi}^{\alpha} Q_{\alpha}$ annihilates the vacuum for a generic spinor parameter $\xi$, the following SWI hold for amplitudes made of $n$ arbitrary fields $\Phi_{i}$ :

$$
\begin{aligned}
0 & =\left\langle 0\left|\left[Q, \mathcal{O}_{n}\right]\right| 0\right\rangle \\
& =\sum_{i}\left\langle 0\left|\Phi_{1} \cdots\left[Q, \Phi_{i}\right] \cdots \Phi_{n}\right| 0\right\rangle,
\end{aligned}
$$

where $\mathcal{O}_{n} \equiv \Phi_{1} \cdots \Phi_{n}$. For a scalar $\phi$ and a Weyl fermion $\psi$ in the same chiral supermultiplet, and a gaugino $\lambda$ and a gauge boson $V$ in the same vector multiplet, one has

$$
\begin{aligned}
{\left[Q(\xi), \lambda^{+}(k)\right] } & =-\theta\langle\xi k\rangle V^{+}(k), \\
{\left[Q(\xi), V^{-}(k)\right] } & =+\theta\langle\xi k\rangle \lambda^{-}(k), \\
{\left[Q(\xi), \phi^{\dagger}(k)\right] } & =-\theta\langle\xi k\rangle \psi^{+}(k), \\
{\left[Q(\xi), \psi^{-}(k)\right] } & =+\theta\langle\xi k\rangle \phi(k) .
\end{aligned}
$$

Equation (B2) holds also for fields with opposite helicity $\pm \rightarrow \mp$ provided one replaces $\phi \leftrightarrow \phi^{\dagger}$ and $\langle\xi k\rangle \rightarrow-[\xi k]$.

For $n=4$, taking $\mathcal{O}_{4}=\lambda_{1}^{+} V_{2}^{+} V_{3}^{+} V_{4}^{+}$in Eq. (B1) gives

$$
\begin{aligned}
0= & \left\langle\xi k_{1}\right\rangle A_{4}\left(V_{1}^{+} V_{2}^{+} V_{3}^{+} V_{4}^{+}\right)+\left[\xi k_{2}\right] A_{4}\left(\lambda_{1}^{+} \lambda_{2}^{+} V_{3}^{+} V_{4}^{+}\right) \\
& +\left[\xi k_{3}\right] A_{4}\left(\lambda_{1}^{+} V_{2}^{+} \lambda_{3}^{+} V_{4}^{+}\right)+\left[\xi k_{4}\right] A_{4}\left(\lambda_{1}^{+} V_{2}^{+} V_{3}^{+} \lambda_{4}^{+}\right) .
\end{aligned}
$$

Since (supersymmetric) gauge interactions conserve helicity, amplitudes involving two gauginos with the same helicity and two gauge fields are vanishing at tree level. Then Eq. (B3) implies $A_{4}\left(V_{1}^{+} V_{2}^{+} V_{3}^{+} V_{4}^{+}\right)=0$. Similarly, by taking $\mathcal{O}_{4}=\lambda_{1}^{+} V_{2}^{-} V_{3}^{+} V_{4}^{+}$in Eq. (B3) and choosing $\xi=k_{1}$ and $\xi=k_{2}$, one obtains $A_{4}\left(V_{1}^{-} V_{2}^{+} V_{3}^{+} V_{4}^{+}\right)=0=$ $A_{4}\left(\lambda_{1}^{+} \lambda_{2}^{-} V_{3}^{+} V_{4}^{+}\right)$. Finally, $\mathcal{O}_{4}=\phi_{1}^{\dagger} \phi_{2} \lambda_{3}^{+} V_{4}^{+}$gives

$$
\begin{aligned}
0= & \left\langle\xi k_{1}\right\rangle A_{4}\left(\psi_{1}^{+} \phi_{2} \lambda_{3}^{+} V_{4}^{+}\right) \\
& -\left[\xi k_{2}\right] A_{4}\left(\phi_{1}^{\dagger} \psi_{2}^{-} \lambda_{3}^{+} V_{4}^{+}\right) \\
& +\left\langle\xi k_{3}\right\rangle A_{4}\left(\phi_{1}^{\dagger} \phi_{2} V_{3}^{+} V_{4}^{+}\right) \\
& +\left[\xi k_{4}\right] A_{4}\left(\phi_{1}^{\dagger} \phi_{2} \lambda_{3}^{+} \lambda_{4}^{+}\right) .
\end{aligned}
$$

The second term in this equation vanishes as a consequence of the $Z_{2}$ chiral symmetry (10). If no cubic scalar term is present in the theory, as we assume, the same symmetry argument also ensures that the last amplitude vanishes. Choosing $\xi=k_{1}$ then implies $A_{4}\left(\phi_{1}^{\dagger} \phi_{2} V_{3}^{+} V_{4}^{+}\right)=0$, while $\xi=k_{3}$ gives $A_{4}\left(\psi_{1}^{+} \phi_{2} \lambda_{3}^{+} V_{4}^{+}\right)=0$.

Since supersymmetry commutes with the SM gauge group $G_{\mathrm{SM}}$ and the color and Lorentz structures factorize in helicity amplitudes, it follows that the above results hold for fermions in generic representations of $G_{\mathrm{SM}}$, and not only for gauginos in the adjoint; this proves Eq. (5). Relations for higher-point amplitudes can be obtained by similar arguments or simply through Eq. (3). Finally, notice that the operators $\psi^{2} \bar{\psi}^{2}, \psi \bar{\psi} \phi^{2} D$ and $\phi^{4} D^{2}$ can be uplifted into a supersymmetric form [41], so that their introduction in the theory does not change the SWI. 
[1] D. Liu, A. Pomarol, R. Rattazzi, and F. Riva, J. High Energy Phys. 11 (2016) 141.

[2] R. Contino, A. Falkowski, F. Goertz, C. Grojean, and F. Riva, J. High Energy Phys. 07 (2016) 144.

[3] ATLAS Collaboration, Phys. Lett. B 753, 69 (2016).

[4] ATLAS Collaboration, Phys. Rev. Lett. 114, 221802 (2015).

[5] CMS Collaboration, Report No. CMS-PAS-SMP-14-016.

[6] CMS Collaboration, Report No. CMS-PAS-FSQ-13-008.

[7] CMS Collaboration, Report No. CMS-PAS-SMP-14-018.

[8] ATLAS Collaboration, Phys. Rev. Lett. 113, 141803 (2014).

[9] L. J. Dixon contribution to D. E. Soper, $Q C D$ and beyond. Proceedings, Theoretical Advanced Study Institute in Elementary Particle Physics, TASI-95, Boulder, 1995 (World Scientific, Singapore, 1996), p. 758; M. L. Mangano and S. J. Parke, Phys. Rep. 200, 301 (1991).

[10] H. Elvang and Y.t. Huang, Scattering Amplitudes, arXiv: 1308.1697 [Cambridge University Press (to be published)].

[11] T. Cohen, H. Elvang, and M. Kiermaier, J. High Energy Phys. 04 (2011) 053.

[12] M. T. Grisaru, H. N. Pendleton, and P. van Nieuwenhuizen, Phys. Rev. D 15, 996 (1977); M. T. Grisaru and H. N. Pendleton, Nucl. Phys. B124, 81 (1977).

[13] S. J. Parke and T. R. Taylor, Phys. Lett. 157B, 81 (1985); Phys. Lett. B 174, 465(E) (1986).

[14] S. J. Parke and T. R. Taylor, Phys. Rev. Lett. 56, 2459 (1986).

[15] F. A. Berends and W. T. Giele, Nucl. Phys. B306, 759 (1988).

[16] B. Grzadkowski, M. Iskrzynski, M. Misiak, and J. Rosiek, J. High Energy Phys. 10 (2010) 085.

[17] G. F. Giudice, C. Grojean, A. Pomarol, and R. Rattazzi, J. High Energy Phys. 06 (2007) 045.

[18] C. Cheung and C. H. Shen, Phys. Rev. Lett. 115, 071601 (2015).

[19] L. J. Dixon, E. W. N. Glover, and V. V. Khoze, J. High Energy Phys. 12 (2004) 015.

[20] E. Witten, Commun. Math. Phys. 252, 189 (2004).

[21] L. J. Dixon and Y. Shadmi, Nucl. Phys. B423, 3 (1994); B452, 724 (1995).
[22] J. Broedel and L. J. Dixon, J. High Energy Phys. 10 (2012) 091.

[23] R. H. Boels and C. Schwinn, Phys. Rev. D 84, 065006 (2011).

[24] F. Coradeschi and P. Lodone, Phys. Rev. D 87, 074026 (2013).

[25] S. D. Badger, E. W. N. Glover, and V. V. Khoze, J. High Energy Phys. 01 (2006) 066.

[26] S. D. Badger, E. W. N. Glover, V. V. Khoze, and P. Svrcek, J. High Energy Phys. 07 (2005) 025.

[27] K. J. Ozeren and W. J. Stirling, Eur. Phys. J. C 48, 159 (2006).

[28] M. S. Chanowitz and M. K. Gaillard, Nucl. Phys. B261, 379 (1985).

[29] A. Wulzer, Nucl. Phys. B885, 97 (2014).

[30] M. Farina, C. Grojean, F. Maltoni, E. Salvioni, and A. Thamm, J. High Energy Phys. 05 (2013) 022.

[31] J. A. Dror, M. Farina, E. Salvioni, and J. Serra, J. High Energy Phys. 01 (2016) 071.

[32] P. L. Cho and E. H. Simmons, Phys. Rev. D 51, 2360 (1995).

[33] C. Degrande, J. M. Gerard, C. Grojean, F. Maltoni, and G. Servant, J. High Energy Phys. 03 (2011) 125.

[34] J. Bramante, A. Delgado, and A. Martin, Phys. Rev. D 89, 093006 (2014).

[35] E. H. Simmons, Phys. Lett. B 226, 132 (1989).

[36] A. Manohar and H. Georgi, Nucl. Phys. B234, 189 (1984).

[37] O. Domenech, A. Pomarol, and J. Serra, Phys. Rev. D 85, 074030 (2012).

[38] A. Biekoetter, A. Knochel, M. Kramer, D. Liu, and F. Riva, Phys. Rev. D 91, 055029 (2015).

[39] R. Contino, C. Grojean, M. Moretti, F. Piccinini, and R. Rattazzi, J. High Energy Phys. 05 (2010) 089.

[40] D. Liu, A. Pomarol, R. Rattazzi, and F. Riva (to be published).

[41] J. Elias-Miro, J. R. Espinosa, and A. Pomarol, Phys. Lett. B 747, 272 (2015). 